\title{
The effect of acarbose on diabetes- and age-related basement membrane thickening in retinal capillaries of the BB/W-rat
}

\author{
Subrata Chakrabarti ${ }^{a}$, P. Varghese Cherian ${ }^{b}$ and Anders A.F. Sima ${ }^{b}$

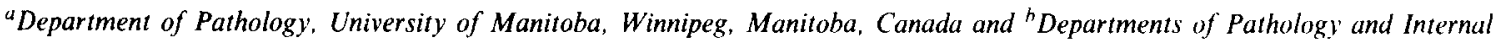 \\ Medicine and the Michigan Diabetes Research and Training Center, University of Michigan, Ann Arbor. MI. USA
}

(Received 16 June 1992: revision accepted 14 November 1992)

\section{Summary}

The effect of the $\alpha$-glucosidase inhibitor acarbose on retinal capillary basement membrane thickening was examined in the spontaneously diabetic BB/W-rat. Four months of diabetes resulted in significant thickening of the basement membranes of both the superficial and deep capillary nets of the retina. This characteristic change of the retinal microvasculature in diabetes was completely prevented by acarbose treatment that substantially reduced postprandial hyperglycemia. A similar but less pronounced effect was seen on the age-rclated increase in basement membrane thickening in acarbose-treated non-diabetic control rats who demonstrated decreased glycated hemoglobin levels compared to non-treated control rats. Significant positive correlations between basement membrane thickness and glycated hemoglobin area suggest that diabetic retinal microangiopathy may be prevented by lowering the cumulative glucose exposure to the microvasculature, and that age-related basement membrane thickening is mediated by long-term exposure to normal glucose levels.

Key words: BB/W-rat; Basement membrane thickening; Retina; Acarbose

\section{Introduction}

Cumulative hyperglycemia is probably the most important risk factor in the development of secondary diabetic complications $[1,2]$. During hyperglycemia glucose levels in the target organs of diabetic complications equilibriate with those of

Correspondence to: Dr. Anders A.F. Sima, Michigan Diabetes Research \& Training Center, University of Michigan, $1331 \mathrm{E}$. Ann Street, Box 0580, Ann Arbor, MI 48109, USA ambient plasma levels, since glucose uptake in these tissues is insulin independent [3]. Lowering of cumulative glucose exposure to these target tissues may therefore ameliorate the metabolic, functional and structural consequences of hyperglycemia [4]. This hypothesis was tested in the present study in which diabetic BB/W-rats were treated with the $\alpha$-sucrose inhibitor acarbose. This compound is a potent competitive inhibitor of small intestine $\alpha$-glucosidase. It delays the generation of monosaccharides in the gut and suppresses 
significantly postprandial elevations in blood glucose and insulin responses in the rat and man [4-7].

We tested the effect of this compound on the characteristic basement membrane thickening (BMT) of the retinal microvasculature in the diabetic BB/W-rat and on the normal age-related increases in $\mathrm{BMT}$ in non-diabetic $\mathrm{BB} / \mathrm{W}$-rats.

\section{Material and Methods}

\section{Animals}

Twelve pre-diabetic male BB/W-rats, and 12 age- and sex-matched non-diabetes-prone $B B / W$ rats were obtained from the National Institutes of Health (NIH) BB-rat colony at the University of Massachusetts (Worcester, MA). At the onset of diabetes, detected by glucosuria (Test Tape, Eli Lilly Canada Inc., Toronto, Ont.), rats were commenced on small daily titrated doses of protamine zinc insulin (Connaught-Novo Inc., Toronto, Ont.) in order to prevent ketoacidosis. Six diabetic and 6 non-diabetic control rats were given free access to rat chow (Lab Blox F-6, Wayne Animal Diets, Winnipeg, Manitoba) and water. The remaining 6 diabetic and 6 control rats were given rat chow mixed with $20 \mathrm{mg}$ acarbose $/ 100 \mathrm{~g}$ rat chow (BAY g 5421, Miles Inc., West Haven, CT) equivalent to a minimal dose of $5 \mathrm{mg} / \mathrm{kg}$ body weight. All rats were kept in air filtered metabolic cages with a 12-h light cycle.

\section{Clinical monitoring}

Body weight, urine volume, and glucosuria were monitored daily. Blood glucose was measured weekly from tail-vein blood samples using an Ames Eyetone (Miles Laboratories Ltd., Rexdale, Ont.). In order to obtain 24-h glucose profiles, untreated and acarbose-treated diabetic rats were monitored as to blood sugar levels every $2 \mathrm{~h}$ for four 24-h cycles. Glycated hemoglobin levels were measured every month using an affinity chromatography test kit (Glyco-Test, Pierce Chemical Co., Rockford, IL). For each individual animal the glycated $\mathrm{Hb}$ area under the curve was calculated as a measure of cumulative hyperglycemia during the observation period. The normal glycated $\mathrm{Hb}$ was arbitrarily set to $100 \%$ and indi- vidual values were expressed as a percentages thereof.

\section{Tissue collection}

Animals were anesthetized with intra-peritoneal injections of Na-pentobarbitol and sacrificed by whole body perfusion with $2.5 \%$ glutaraldehyde in $0.1 \mathrm{M} \mathrm{Na}$-cacodylate buffer ( $\mathrm{pH} 7.4$ ). From the left eye the posterior eyecup was dissected within 3 $\mathrm{mm}$ of the optic nerve head and fixed in the same fixative for $4 \mathrm{~h}$ at $4^{\circ} \mathrm{C}$. The tissues were post-fixed in Na-cacodylate buffered $1 \%$ osmic tetroxide ( $\mathrm{pH}$ 7.4) for $2 \mathrm{~h}$ at $4^{\circ} \mathrm{C}$, dehydrated and embedded in Epon. Ultrathin sections cut perpendicular to the retinal surface were stained with aqueous uranyl acetate and lead citrate and examined electron microscopically as previously described [8].

\section{Morphometric techniques}

Electronmicrographs with a total magnification of 25000 times were used for the capillary basement membrane (BM) measurements. From each retina 10 randomly selected, transversely sectioned capillaries from each the superficial and the deep capillary beds were investigated. BMT was measured with the aid of a Hewlett Packard 9874A digitizer interfaced with a Hewlett Packard 9875A computer (Hewlett Packard, Fort Collins, CO) as previously described in detail [9]. Briefly, the total capillary area was measured, BM area was determined by subtracting lumenal, endothelial cell. and pericyte areas from the total area. Mean BM length was calculated by measuring the lengths of the lines delineating the BM divided in half. BMT was expressed as area per unit length (nm). This method takes into account the variability of the BM contour and gives an average value of BMT.

\section{Statistical analysis}

The data are expressed as means \pm S.E.M. and were analyzed by one-way analysis of variance and linear contrast. Linear regression analyses were performed using the least square method.

\section{Results}

\section{Clinical observations}

Acarbose-treated and non-treated diabetic rats 


\section{TABLE 1}

Comparisons of body weight, blood glucose, glycated hemoglobin, glycated hemoglobin area, and basement membrane thickness in the two capillary nets between acarbose-treated diabetic and control rats, and non-treated diabetic and control rats ${ }^{\mathrm{a}}$

\begin{tabular}{|c|c|c|c|c|c|c|}
\hline & \multirow[b]{2}{*}{$\begin{array}{l}\text { Body weight } \\
\text { (g) }\end{array}$} & \multirow[b]{2}{*}{$\begin{array}{l}\text { Blood glucose } \\
(\mathrm{mmol})\end{array}$} & \multirow[b]{2}{*}{$\begin{array}{l}\text { Glycated } \mathrm{Hb} \\
(\%)\end{array}$} & \multirow[b]{2}{*}{$\begin{array}{l}\text { Glycated } \mathrm{Hb} \\
\text { area } \\
(\% \text { of normal) }\end{array}$} & \multicolumn{2}{|c|}{$\begin{array}{l}\text { Basement membrane thickness } \\
(\mathrm{nm})\end{array}$} \\
\hline & & & & & $\begin{array}{l}\text { Superficial } \\
\text { capillary } \\
\text { bed }\end{array}$ & $\begin{array}{l}\text { Deep capillary } \\
\text { bed }\end{array}$ \\
\hline $\begin{array}{l}\text { Non-treated } \\
\text { control rats } \\
(n=6)\end{array}$ & $457+7$ & $4.7 \pm 0.6$ & $4.5 \pm 0.4^{\mathrm{e}}$ & $100.0 \pm 4.5^{\mathrm{g}}$ & $122.7 \pm 5.2^{\mathrm{h}}$ & $72.0 \pm 2.8^{h}$ \\
\hline $\begin{array}{l}\text { Acarbose-treated } \\
\text { control rats } \\
(n=6)\end{array}$ & $439 \pm 9$ & $5.6 \pm 0.4$ & $3.8 \pm 0.2$ & $73.1 \pm 2.4$ & $92.6 \pm 6.2^{i}$ & $57.9 \pm 2.3^{i}$ \\
\hline $\begin{array}{l}\text { Non-treated } \\
\text { diabetic rats } \\
(n=6)\end{array}$ & $365 \pm 6^{b}$ & $17.8 \pm 3.0^{\mathrm{b}}$ & $9.8 \pm 0.6^{\mathrm{c} . f}$ & $160.7 \pm 3.4^{b . d}$ & $177.6 \pm 5.5^{b, d}$ & $105.1 \pm 4.1^{b, d}$ \\
\hline $\begin{array}{l}\text { Acarbose-treated } \\
\text { diabetic rats } \\
(n=6)\end{array}$ & $383 \pm 13^{b}$ & $13.8 \pm 0.6^{b}$ & $7.2 \pm 0.3^{c}$ & $127.9 \pm 2.9^{c}$ & $113.0 \pm 6.1$ & $72.4 \pm 6.8$ \\
\hline
\end{tabular}

a Values are means \pm S.E.M. of $n=6$ in each group.

${ }^{b} P<0.001$ comparcd to non-treated control rats or acarbose-treated control rats.

' $P<0.001$ compared to acarbose-treated control rats.

${ }^{d} P<0.001$ compared to acarbose-treated diabetic rats.

${ }^{c} P<0.01$ compared to non-treated diabetic rats or acarbose-treated diabetic rats.

f $P<0.05$ compared to acarbose-treated diabetic rats.

${ }^{\mathrm{B}} P<0.001$ compared to acarbose-treated control rats.

${ }^{\text {h }} P<0.05$ compared to acarbose-treated control rats.

i $P<0.02$ compared to acarbose-treated diabetic rats.

i $P<0.05$ compared to acarbose-treated diabetic rats.

showed hyperglycemia, elevated glycated hemoglobin levels, and decreased body weights when compared to treated and non-treated control rats (Table 1). Although single blood glucose measurements at 15:00 $\mathrm{h}$ did not reveal any significant differences between acarbose treated and non-treated diabetic rats (Table 1), the $24 \mathrm{~h}$ blood glucose area in acarbose-treated diabetic rats was significantly decreased by $40 \%$ as previously reported [4]. This decrease was reflected in a significant $(P<0.001)$ lowering of the glycated hemoglobin area during the study period in acarbose-treated diabetic rats (Table 1 and Fig. 1). Acarbose-treatment of non-diabetic control rats resulted in a $27 \%$ significant $(P<0.001)$ decrease in glycated hemoglobin area as compared to nontreated control rats (Table 1 and Fig. 1). Acarbose treatment was well tolerated by treated animals who showed no weight loss or increased frequency of diarrhea.

\section{Effect on BMT}

The BMT of both the superficial and deep capillary nets were significantly increased in nontreated diabetic BB/W-rats (Table 1 and Fig. 2). This increase in BMT was completely prevented by acarbose-treatment of diabetic rats (Table 1 and Fig. 2). In control rats acarbose-treatment had a small but statistically significant $(P<0.05)$ effect on the presumably age-related BM thickening [10] (Table 1). Highly significant correlations were obtained between glycated hemoglobin areas and BMT of the superficial and the deep capillary net (Fig. 3). 


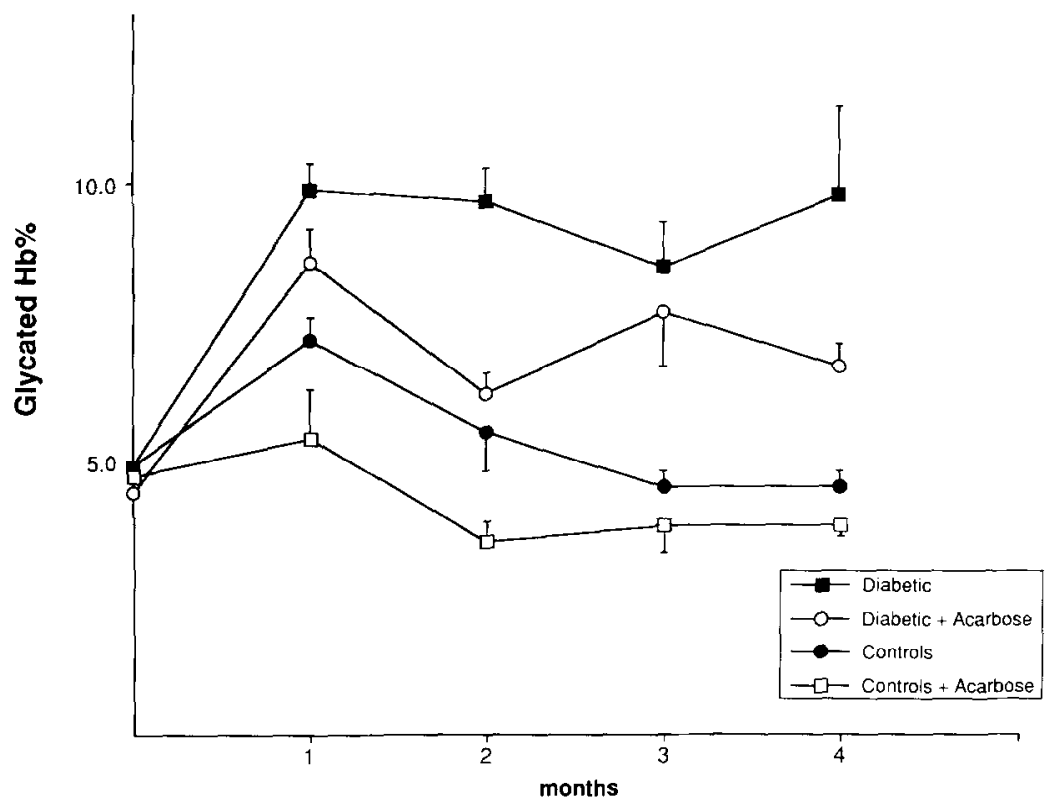

Fig. 1. As a measure of cumulative hyperglycemia, the areas under the curve formed by monthly glycated Hb-measurements was employed. $\mathbf{\square}$, diabetic rats, $n=6 ; 0$, acarbose-treated diabetic rats, $n=6 ; 0$. control rats, $n=6 ; 0$, acarbose-treated control rats, $n=6$.

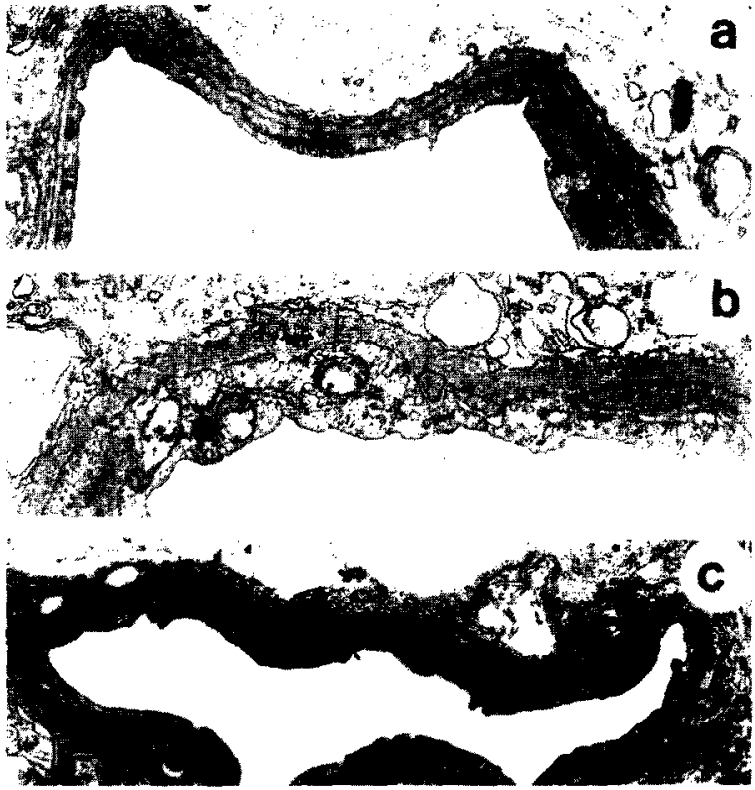

Fig. 2. Electronmicrographs comparing BMT in the deep capillary net in (a) non-treated control rat, (b) non-treated diabetic rat, and (c) acarbose-treated diabetic rat. Mag. $\times 25000$.

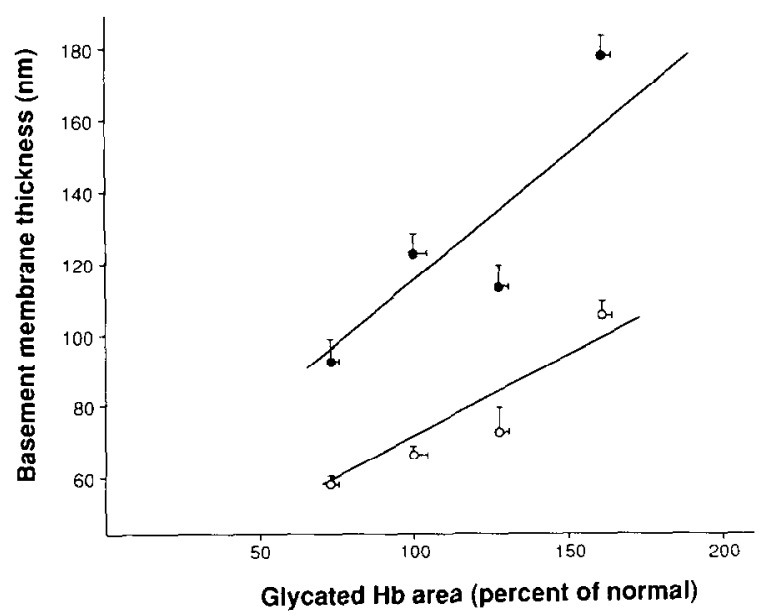

Fig. 3. Regression analyses between glycated $\mathrm{Hb}$ area and BMT in the superficial capillary net $(0)$ and the BMT of the capillaries in the deep net (O). The points are group means \pm S.E.M. and each point represents 6 animals. Regression for the superficial BMT showed: $r=0.69, n=24, P<$ 0.001 ; for the deep capillary BMT: $r=0.78, n=24, P<0.001$. 


\section{Discussion}

In the present communication we have demonstrated that successful lowering of postprandial hyperglycemia resulting in decreased cumulative hyperglycemia in diabetic $\mathrm{BB} / \mathrm{W}$ rats prevents the characteristic BMT in retinal capillaries. The pathophysiology of BMT in diabetes and aging is not known in detail [11]. It has been suggested that non-enzymatic glycation of extracellular matrix constituents such as various collagen species and fibronectin may play a major role in altering the physiochemical and permeability properties of BMs [12,13]. Decreased synthesis of proteoglycans and subsequent deprivation of anionic sites probably affects the charge selective BM barrier function [14] and is accompanied by an enhanced collagen IV gene expression and increased production of matrix proteins $[15,16]$. However, fructose which is secondarily elevated in diabetes via the polyol pathway may be more important than glucose itself in explaining BMT. Fructose possesses a multifold higher affinity to bind non-enzymatically to proteins and promote subsequent cross-linkages [17-19]. The fact that the polyol pathway is localized to both endothelial cells and pericytes of retinal capillaries [20] and that aldose reductase inhibition prevents BMT in retinal capillaries in the presence of hyperglycemia lends further credence to this notion $[8,21,22]$. Hence, the effect of acarbose on retinal capillary BMT could be mediated by a decreased cumulative exposure of the microvasculature to glucose per se, and/or by lowered local fructose concentrations mediated by a decreased activity of the polyol-pathway secondary to decreased hyperglycemia.

Structural abnormalities characteristic of diabetic neuropathy which are more closely linked to the activation of the polyol-pathway are significantly ameliorated following acarbose treatment [4], suggesting that this pathway is indeed deactivated by the present treatment regimen. It is conceivable that elimination of pronounced postprandial hyperglycemia by acarbose [4] is sufficient to inhibit the induction of aldose reductase [23], despite the fact the cumulative hyperglycemia in acarbose treated diabetic rats exceeded that of non-diabetic control rats.
An unexpected finding in the present study was the significant attenuation of the age-related BMT in acarbose-treated non-diabetic control rats most likely due to decreased ambient glucose levels as reflected by a significant decrease in glycated hemoglobin area compared to non-treated control rats. It is unlikely that other nutritional factors would be responsible for this finding, since acarbose-treated control rats showed no weight loss or increased frequency of diarrhea compared to non-treated control rats.

In summary the present findings suggest that suppression of the cumulative glucose load has an attenuating effect on the development of the characteristic BMT of retinal capillaries associated with diabetes and normal aging.

\section{Acknowledgements}

This study was supported in part by grants from Miles Inc., New Haven, CT, the Canadian Medical Research Council (MT-I 0673, MT-10674) and NIH (DK-38304). The authors are thankful to Ms. Elaine Moore for preparing the manuscript.

\section{References}

1 Pirart, J. (1978) Diabetes mellitus and its degenerative complications: a prospective study of 4,400 patients observed between 1947 and 1973. Diabetes Care 1, 168-188.

2 Maser, R.E., Steenkiste, A.R., Dorman, J.S. et al. (1989) Epidemiological correlates of diabetic neuropathy. Report from Pittsburgh Epidemiology of Diabetes Complications Study. Diabetes 38, 1456-1461.

3 Winegrad, A.I. (1987) Does a common mechanism induce the diverse complications of diabetes? Diabetes 36 , 396-406.

4 Sima, A.A.F. and Chakrabarti. S. (1992) Long-term suppression of post-prandial hyperglycemia with acarbose retards the development of neuropathies in the BB/W-rat. Diabetologia 35, 325-330.

5 Gray, R.S. and Olefsky, J.M. (1982) Effect of glucosidase inhibitor on the metabolic response of diabetic rats to a high carbohydrate diet consisting of starch and sucrose, or glucose. Metabolism 31, 88-92.

6 Yamashita, K., Sugawara, S. and Sakairi, I. (1984) Effects of an alpha-glucosidase inhibitor. Acarbose, on blood glucose and serum lipids in streptozotocin-induced diabetic rats. Horm. Metab. Res. 16, 179-182.

7 Hillebrand, I., Boehm, K., Graefe, K.H. and Wehling, K. 
(1986) The effect of a new $\alpha$-glucosidase inhibitors (BAY in 1099 and BAY o 1248) on meal-stimulated increases in glucose and insulin levels in man. Klin. Wochenschr. 64, 393-396.

8 Chakrabarti, S. and Sima, A.A.F. (1989) Effect of aldose reductase inhibition and insulin treatment on retinal capillary basement membrane thickening in BB-rats. Diabetes 38, 1181-1186.

9 McEwen, T.A.J., Chakrabarti, S. and Sima, A.A.F. (1987) A rapid reproducible method for determination of basement membrane thickness in biological structures. Comput. Biol. Med. 14, 586-592.

10 Sima, A.A.F., Chakrabarti, S., Garcia-Salinas, R. and Basu, P.K. (1985) The BB-rat - an authentic model of human diabetic retinopathy. Curr. Eye Res. 4, 1087-1092.

11 Williamson, J.R., Tilton, R.G., Chang, K. and Kilo, C. (1988) Basement membrane abnormalities in diabetes mellitus: Relationship to clinical microangiopathy. Diab. Metab. Rev. 4, 339-370.

12 Vlassara, H., Brownlee, M. and Cerami, A. (1986) Nonenzymatic glycosylation: Role in the pathogenesis of diabetic complications. Clin. Chem. 32, B37-B41.

13 Cohen, M.P. (1986) Diabetes and protein glycosylation. Measurement and biologic relevance, Chapter 5, Springer Verlag, New York, pp. 67-111.

14 Chakrabarti, S., Ma, N. and Sima, A.A.F. (1991) Anionic sites in diabetic basement membranes and their possible role in diffusion barrier abnormalities in the BB-rat. Diabetologia 34, 301-306.

15 King, G.L., Berman, A. and Ledbetter, S. (1988) Regulation of type IV collagen mRNA in retinal capillary endothelial cells and pericytes by glucose levels and aldose inhibitor. Invest. Ophthalmol. Vis. Sci. 29 (suppl.), 181.
16 Ledbetter, S., Copeland, E.J., Noonan, D., Vogeli, G. and Hassell, J.R. (1990) Altered steady state mRNA levels of basement membrane proteins in diabetic mouse kidneys and thromboxane synthase inhibition. Diabetes 39 . 196-203.

17 McPherson, J.D., Shilton, B.H. and Walton, D.J. (1988) Role of frucose in glycation and cross-linking of proteins. Biochemistry 27, 1901-1907.

18 Walton, D.J., McPherson, J.D. and Shilton, B.H. (1989) Fructose mediated crosslinking of proteins. Prog. Clin. Biol. Res. 304, 163-i70.

19 Grandhee, S.K., Sell, D.R. and Monnier, V.M. (1991) Mechanism of formation of the tissue matrix crosslink pentosidine. Diabetes 40 (suppl. 1), 203A.

20 Chakrabarti, S., Sima, A.A.F., Nakajima, S., Yagihashi, S. and Greene, D.A. (1987) Aldose reductase in the BBrat. Isolation, immunological identification and localization in the retina and peripheral nerve. Diabetologia 30 , 244-251.

21 Sima, A.A.F., Chakrabarti, S., Tze, W.J. and Tai, J. (1988) Pancreatic islet allograft prevents basement membrane thickening in the diabetic retina. Diabetologia 31, 175-181.

22 Robison, W.G. Jr., Kador, P.F., Akagi, Y., Kinoshita, J.H., Gonzalez, R. and Dvornik, D. (1986) Prevention of basement membrane thickening in retinal capillaries by a novel inhibitor of aldose reductase. Tolrestat. Diabetes 35, 295-299.

23 Ghahary A., Chakrabarti, S., Murphy, L.J. and Sima, A.A.F. (1991) Effect of insulin and statil on aldose reductase expression in diabetic rats. Diabetes 40, 1391-1396. 